\title{
Health professions education in pandemics and epidemics: A proposed framework for educators
}

\section{Dear Editor,}

The COVID-19 pandemic has disrupted healthcare systems and health professions education (HPE). There are few frameworks to help educators manage HPE before, during and after pandemics and epidemics. We developed a crisis management framework which draws from diverse theories to emphasise preparedness, leadership, stakeholder perceptions and organisational learning to provide guidance. ${ }^{1}$ Bundy et al. define crisis management in 3 stages: before, during and after a crisis. This allows detailed response planning. ${ }^{1} \mathrm{We}$ adapted it to derive a framework for HPE in pandemics and epidemics (HPEPE). This framework is relevant as it reminds educators to not only focus on the present pandemic, but also the steps to take before and after the crisis to learn and prepare further for the future.

We performed a systematic literature review and identified 208 publications relevant to HPEPE. We distilled the key points, grouped them into 6 elements, reaffirmed representativeness, then mapped them back to the crisis management framework. ${ }^{1}$ We describe 6 elements, comprising $6 \mathrm{Cs}$-curriculum, continuing professional development (CPD), communications, courage, communities and continuity-and demonstrate their relevance to HPEPE and crisis management stages. Table 1 illustrates how our proposed framework was applied during the COVID-19 pandemic in the Singapore context.

Curriculum. Swift curriculum adaptation is essential in HPEPE..$^{2-4}$ Hybrid models, combining remote and onsite teaching and training could be adopted. Similarly, to minimise risk of exposure, alternatives could be used for assessments, such as electronic proctoring for written examinations, or use of simulated patients and videoconferencing for clinical assessments. Virtual selection interviews and even graduation ceremonies could be conducted online. Investing in technology resources (high-speed Internet access, video-production facilities, videoconferencing software and learning management systems ${ }^{5}$ ) and upskilling faculty to thoughtfully use technology ${ }^{5}$ are crucial for HPEPE. While technological innovations are welcome, educational principles should guide design and implementation, ${ }^{5}$ with emphasis placed on programme evaluation. Context must be considered during implementation. Uptake of technology varies depending on resource settings, ${ }^{3}$ and increased technology use may exacerbate resource disparities between learners, regions or countries, leading to disenfranchised learners. Education systems should thus provide additional resources as appropriate.

During pandemics, important but less visible curriculum elements (including leadership skills, ethics, decision-making in uncertainty, resource management, adaptability and professionalism) may be opportunistically and authentically taught using case discussions, reflection and role modelling., ${ }^{6,7}$ Postpandemic, schools should re-examine the teaching and integration of public health, epidemiology and infection control preparedness ${ }^{6,7}$ in their curricula during curricular review.

CPD. During pandemics, adapting CPD for healthcare professionals is vital for just-in-time learning and up-to-date practice as pandemic-related medical knowledge evolves rapidly. ${ }^{3}$ Frontline healthcare professionals should educate themselves through trustworthy CPD resources and international resources (e.g. online resources by the US Centers for Disease Control and Prevention and the World Health Organization) for accessible and up-to-date information. Post-pandemic CPD might embrace a wider range of pandemic-related topics such as communications, technology-enhanced learning, disaster medicine, or psychological effects of the pandemic on individuals or societies.

Communications. Good communications applies equally to crisis management ${ }^{1}$ and HPEPE. Communications is important in all stages of HPEPE, but particularly critical during the pandemic for internal and external stakeholders. ${ }^{6,8}$ Two-way communications for internal stakeholders (learners, faculty and administrators) serve to provide information, receive feedback, offer reassurance and maintain morale; technology can extend the reach of these communications. Communications allow engagement, build trust and may mitigate the sense of isolation. For external stakeholders (healthcare institutions or government/regulatory bodies), communications allow for effective coordination of student/resident placements, planning of curricular changes and crafting of education-related policies. Before the next pandemic, planning for defined communications protocols and teams can be done. ${ }^{2}$ 


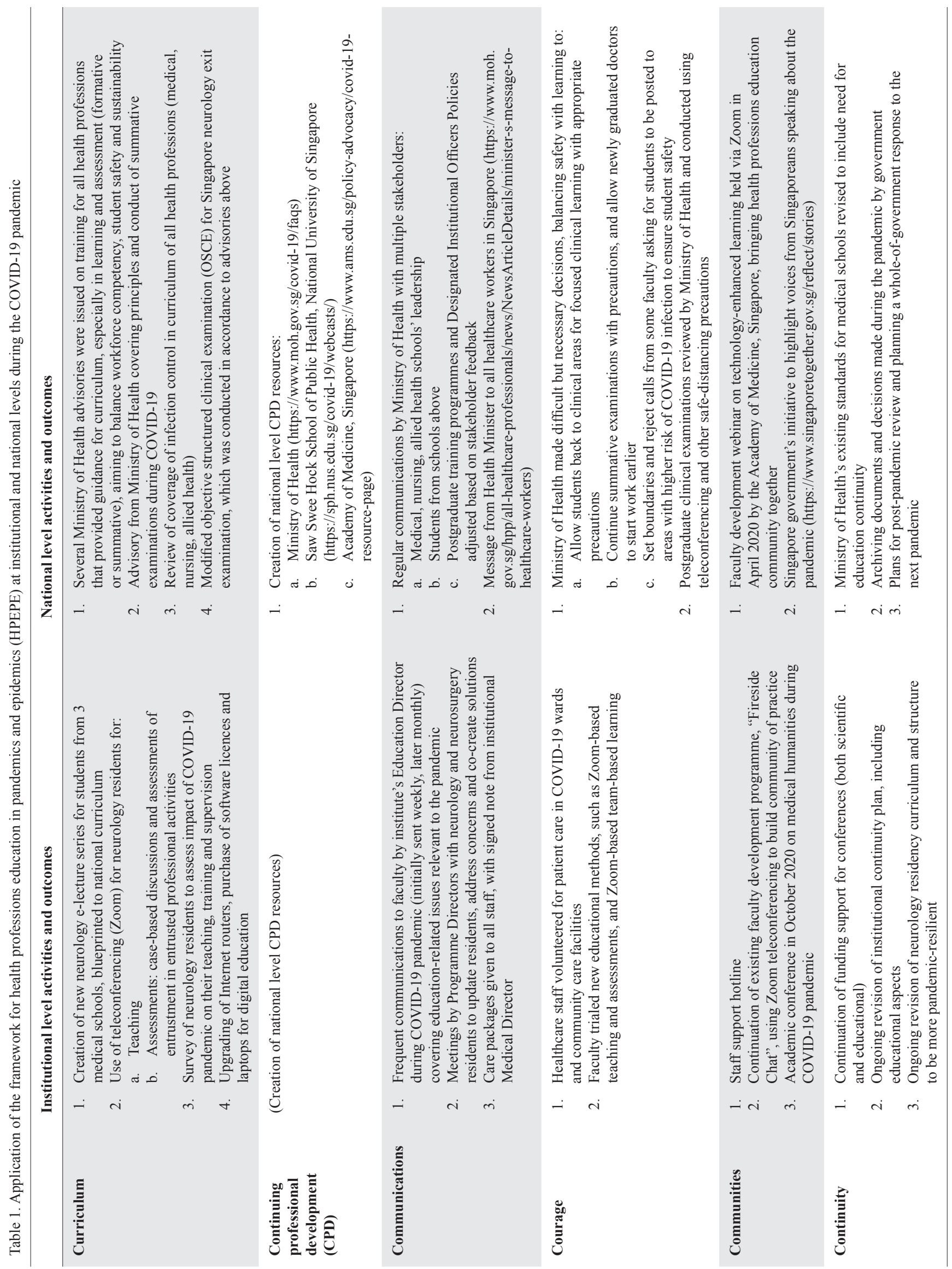


Courage. This is important in all stages of a pandemic. Courageous behaviours take many forms and can be displayed by both individuals and organisations. ${ }^{7}$ As individuals, faculty members become role models by courageously staying true to their ethical duty to provide care. Such behaviours reinforce professional values for junior learners and facilitate the formation of professional identity. In bravely embracing lifelong learning, faculty can learn new technology skills, while students/residents can adopt new roles and responsibilities by becoming peer teachers or preparing CPD updates.

As organisations, schools and training programmes need to restructure curricular components and make difficult curricular decisions during pandemics. Courageous leadership is needed to make necessary sacrifices. Schools may need to graduate students earlier to supplement the workforce. ${ }^{6}$ This requires strong leadership to address student/parental concerns, while making the necessary curricular adaptations to ensure that critical competencies are achieved before graduation. Post-pandemic reviews are also important to courageously acknowledge failures and also celebrate successes. Key learning points should be documented.

Communities. During and after pandemics, our learners experience fear and isolation. Healthcare communities can provide much-needed emotional support to learners. ${ }^{7}$ The educator community can also support learners using a variety of approaches to encourage self-care, constructivist growth and professional development. ${ }^{9}$ Our learners, as fledgling members of a healthcare community of practice, ${ }^{4}$ can assist in clinical care during pandemics. This facilitates acculturation into the community of practice via legitimate peripheral participation that forms their professional identity.

Continuity. The crisis management literature emphasises the utility of business continuity plans. As an example, higher education maintains analogous academic continuity plans that can be adopted and developed for HPE systems. ${ }^{8,10}$ A coordinating taskforce should be formed once the crisis is recognised to ensure continuity of the education mission, which is contingent upon the first 5 elements in our framework. If additional resources are needed to support these preceding elements, organisations should acquire them as a demonstrated commitment to education continuity. Post-pandemic, the continuity plans should be refined after review, and can be also stress-tested and strengthened via simulation.
Our proposed HPEPE framework emphasises principles and critical influences. As it is new, it has yet to be successfully implemented in full throughout all stages of a pandemic. We also acknowledge that the framework has only been used in a healthcare system in Singapore. Therefore, context needs to be considered in implementation. Future studies on application of this framework will help with validation and refinement.

Epidemics and pandemics will undoubtedly recur. A framework will help educators manage these crises, and plan responses to current and future crises. In this way, HPE becomes an active enabler of learning and resilience for students, faculty, and the health and education systems.

\section{REFERENCES}

1. Bundy J, Pfarrer MD, Short CE, et al. Crises and Crisis Management: Integration, Interpretation, and Research Development. J Manag 2017;43:1661-92.

2. Samarasekera DD, Goh DLM, Lau TC. Medical School Approach to Manage the Current COVID-19 Crisis. Acad Med 2020;95:1126-7.

3. Berwick DM. Choices for the "New Normal". JAMA 2020:2125-6.

4. Cleland J, McKimm J, Fuller R, et al. Adapting to the impact of COVID-19: Sharing stories, sharing practice. Med Teach 2020; 42:772-5.

5. Sandars J, Correia R, Dankbaar M, et al. Twelve tips for rapidly migrating to online learning during the COVID-19 pandemic. MedEdPublish 2020;9:82.

6. Lucey CR, Johnston SC. The Transformational Effects of COVID-19 on Medical Education. JAMA 2020;324:1033-4.

7. Sklar DP. COVID-19: Lessons From the Disaster That Can Improve Health Professions Education. Acad Med 2020;95:1631-3.

8. Ashokka B, Ong SY, Tay KH, et al. Coordinated responses of academic medical centres to pandemics: Sustaining medical education during COVID-19. Med Teach 2020:42:762-71.

9. Wald HS. Optimizing resilience and wellbeing for healthcare professions trainees and healthcare professionals during public health crises - Practical tips for an 'integrative resilience' approach. Med Teach 2020:42:744-55.

10. Regehr C, Nelson S, Hildyard A. Academic continuity planning in higher education. J Bus Contin Emer Plan 2017;11:73-84.

Nigel Choon Kiat Tan ${ }^{1}{ }_{F R C P}$,

Mabel Anne Mei Poh $\underline{\operatorname{Yap}}^{2}{ }^{2}$,, Kevin $\underline{\operatorname{Tan}}{ }^{1}$ MRCP

${ }^{1}$ Office of Neurological Education, Department of Neurology, National Neuroscience Institute, Singapore

${ }^{2}$ Professional Training and Assessment Standards Division, Ministry of Health, Singapore

Correspondence: A/Prof Kevin Tan, Office of Neurological Education, Department of Neurology, National Neuroscience Institute, 11 Jalan Tan Tock Seng, Singapore 308433

Email: kevin.tan@singhealth.com.sg 